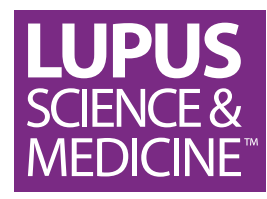

\title{
Lupus clinical trial eligibility in a real- world setting: results from the British Isles Lupus Assessment Group- Biologics Register (BILAG-BR)
}

To cite: Dyball S, Collinson S, Sutton $\mathrm{E}$, et al. Lupus clinical trial eligibility in a real-world setting: results from the British Isles Lupus Assessment GroupBiologics Register (BILAG-BR). Lupus Science \& Medicine 2021;8:e00513. doi:10.1136/ lupus-2021-000513

- Additional supplemental material is published online only. To view, please visit the journal online (http://dx.doi.org/10. 1136/lupus-2021-000513).

Received 28 April 2021 Accepted 13 July 2021

Check for updates

(C) Author(s) (or their employer(s)) 2021. Re-use permitted under CC BY-NC. No commercial re-use. See rights and permissions. Published by BMJ.

${ }^{1}$ Centre for Epidemiology Versus Arthritis, Division of Musculoskeletal \& Dermatological Sciences, The University of Manchester Faculty of Biology Medicine and Health,

Manchester, UK

${ }^{2}$ The Kellgren Centre for Rheumatology, NIHR Manchester Musculoskeletal Biomedical Research Unit, Central Manchester University Hospitals NHS Foundation Trust, Manchester, UK

Correspondence to

Dr Ben Parker; ben.parker@mft. nhs.uk

\section{ABSTRACT \\ Objective To quantify how well phase III randomised clinical trials in both SLE and lupus nephritis (LN) represents a real-world SLE cohort.}

Methods Literature reviews were performed of major published phase III SLE $(n=12)$ and $L N(n=6)$ clinical trials ( ClinicalTrials.gov). Inclusion and exclusion criteria common across these trials were collated for non-renal SLE or $\mathrm{LN}$ trials, and applied to patients recruited to the British Isles Lupus Assessment Group-Biologics Register (BILAG$\mathrm{BR}$ ) starting either biological or standard-of-care (SOC) therapies.

Results We recruited 837 patients to the BILAG-BR from September 2010 to June 2018, starting either SOC $(n=125,15 \%)$ or a biological medication $(n=712,85 \%)$. Active LN, defined as a BILAG A in the renal domain occurred in 20\% ( $n=166)$. Overall, $530(63 \%)$ patients were ineligible to participate in non-renal SLE clinical trials and $72(43 \%)$ patients with active LN would be ineligible for $L N$ trials. The most common reasons for ineligibility from the non-renal lupus trials included active renal involvement $(n=166,20 \%)$ and low disease activity $(n=114,15 \%)$. For $L N$ trials, the most common exclusion met was pre-existing renal impairment $(n=15,9 \%)$. Patients with fewer comorbidities were more likely to be eligible to participate in non-renal SLE trials.

Conclusions In this national register of patients with moderate-to-severe SLE, nearly two-thirds would not be eligible for recruitment to key SLE clinical trials nor would almost half of those with active LN. Eligibility criteria may excessively constrain enrolment and thus, how we can generalise trial results in a real-world setting.

\section{BACKGROUND}

SLE is a chronic multisystem autoimmune disorder which has diverse manifestations that can range from a mild rash or arthritis to severe organ-threatening disease. $\mathrm{LN}$ is a severe complication of SLE and a major cause of overall morbidity and mortality with up to $30 \%$ of patients with LN progressing to endstage renal failure. ${ }^{1-3}$

\section{Key messages}

What is already known about this subject?

- There is a high volume of clinical trials in SLE, with many trials reporting insufficient patient recruitment as a reason for termination.

What does this study add?

- In this cohort of moderate-to-severe SLE, almost two-thirds of patients would not be eligible for phase III SLE clinical trial participation, and almost a half of all patients with active renal lupus would be excluded from phase III lupus nephritis (LN) trials.

- Patients excluded from non-renal SLE trials were more likely to be multimorbid.

How might this impact on clinical practice or future developments?

- Due to high numbers of patients excluded, the results from phase III clinical trials may not be generalisable to the real-world SLE population.

- We would recommend that phase III clinical trial exclusion criteria should be minimised where possible to maximise recruitment potential, speed their conduct and increase their external validity.

Conventional immunosuppressant medications and corticosteroids represent the standard of care (SOC) in SLE management, however, they can be associated with a range of adverse effects and are not always effective. Despite a recent series of successful trials in SLE including belimumab (BLISS-LN, BLISS-52, BLISS-76), anifrolumab (TULIP 2) and vorisporin (AURORA), a major challenge in caring for patients with SLE is the paucity of approved medications with several randomised controlled trials (RCTs) proving unsuccessful in achieving their primary end points in SLE including epratuzumab (EMBODY1/2), tabalumab (ILLUMINATE 1/2) and anifrolumab (TULIP 1). ${ }^{4-12}$

RCTs represent the gold standard to determine initial efficacy and safety of medicines, 
using idealised and controlled circumstances; realworld registry data provide additional data on long-term follow-up in a broader population. Trials that focus on LN have the advantage of objective hard end points, such as protein-creatinine ratios and serum creatinine concentrations, whereas non-renal SLE trials have no clear biomarker of treatment response and as such are dependent on more subjective outcome measures such as composite indices of disease activity (eg, SLE Disease Activity Index, SLE Responder Index-4, etc). By convention, SLE clinical trials are usually divided into renal (LN) and non-renal trials.

There are complex reasons why clinical trials fail to meet their primary end points in SLE. ${ }^{13}$ Stringent inclusion and exclusion criteria are often applied to create a more homogenous disease group such as antibody positivity, involvement of specific organ systems, mandating a severity level of disease activity and use of SLE classification criteria to define the disease population. Effective SOC therapies including high-dose corticosteroids and concomitant prescribing of immunosuppressive therapies may also confuse the interpretation of trial results. Other common exclusion criteria in clinical trials include organ dysfunction or comorbidities as there may be concern that these may be incorrectly attributed to the trial medication. This may however, reduce the external validity of a clinical trial, rendering them less applicable to the intended patient population. Our aim was to quantify how accurately phase III blinded RCTs in both SLE and LN represent a real-world SLE cohort of patients with moderate-to-severe disease.

\section{METHODS}

\section{Identification of eligible clinical studies}

We searched on the 'ClinicalTrials.gov' database which records privately and publicly funded clinical studies conducted around the world in August 2020, first using the search term "SLE" or "systemic lupus erythematosus" and second "lupus nephritis" to identify relevant studies. In the search, we included phase III, double-blinded, RCTs involving patients with SLE, who were treated by either a biological therapy or a targeted synthetic diseasemodifying antirheumatic drug. The study must have completed recruitment; however, results did not have to be formally published. We excluded trials that published data on extended follow-up of previously published RCTs, open-label studies or studies not deemed to be the principle study, such as those using an alternative method of administration or different study population in a previously trialled medication.

\section{Patients and study design}

The British Isles Lupus Assessment Group-Biologics Register (BILAG-BR) is a national, multicentre, prospective cohort study. It recruits patients with a diagnosis of SLE that are $\geq 5$ years old and can provide informed consent. There are two arms to the BILAG-BR, a biologics cohort who are commencing or restarting biological therapy, and a SOC cohort who are newly receiving an immunosuppressant medication (including mycophenolate, azathioprine, ciclosporin, cyclophosphamide or tacrolimus) and are biologically naïve. Patients accessing a biological therapy for SLE in the UK are required to meet strict eligibility guidelines, based either on high disease activity (eg, 1 BILAG A, 2×BILAG B, SLE Disease Activity Index (SLEDAI) $>6$ ) or the need for unacceptably high doses of steroids despite optimal SOC.

Patients starting either a biological or SOC therapy were included in this cross-sectional analysis at cohort entry. Demographic details including age, disease duration, sex and race/ethnicity were noted, along with baseline laboratory results, past and current therapies and disease activity. SLE disease activity was measured by the BILAG-2004 index and SLEDAI 2000 (SLEDAI-2K). ${ }^{14} 15$ Data were collected on common comorbidities for all patients at registration in the BILAG-BR.

\section{Non-renal lupus inclusion and exclusion criteria}

We applied available data to common inclusion criteria including age $\geq 18$ years, positive for American College of Rheumatology (ACR)-1997 SLE classification criteria, ${ }^{16}$ positive serology anti-double stranded DNA (dsDNA) or ANA antibodies (in trials these are performed in a central laboratory, for this study we recorded the antibody status at entry or within 6 months of study enrolment), and moderate-to-severe active disease, defined as a BILAG A in 1 domain or a BILAG $\mathrm{B}$ in $\geq 2$ domains, or a SLEDAI-2K $\geq 6$.

Exclusion criteria common across multiple clinical trials included steroids equivalent to $>40 \mathrm{mg}$ prednisolone daily, active renal or neurological lupus (defined as a BILAG A in either domain) and a urinary proteincreatinine ratio (uPCR) $\geq 100 \mathrm{mg} / \mathrm{mmol}$, a history of viral hepatitis B or C, a history of malignancy (excluding basal cell carcinoma), cyclophosphamide within 90 days, B-cell therapy within the previous year, CKD stage 4 or 5 and low blood counts (defined as neutrophils $<1.0 \times 10^{9} / \mathrm{L}$, platelets $<10 \times 10^{9} / \mathrm{L}$ or $\left.\mathrm{Hb}<70 \mathrm{~g} / \mathrm{L}\right)$.

\section{LN inclusion and exclusion criteria}

Active LN was defined as a BILAG score A in the renal domain. The inclusion criteria common across majority of LN trials included age $\geq 18$ years, meeting ACR-1997 SLE classification criteria, positive anti-dsDNA or ANA antibodies (see above) and proteinuria (defined as a uPCR $>100 \mathrm{mg} / \mathrm{mmol}$ ).

Common exclusion criteria in renal trials included active neurological lupus (defined as a BILAG A central nervous system (CNS) domain), a history of viral hepatitis $\mathrm{B}$ or $\mathrm{C}$, a history of malignancy (excluding basal cell carcinoma), cyclophosphamide within 90 days, B-cell therapy within the previous year, chronic kidney disease (CKD) stage 5 and low blood counts (defined as neutrophils $<1.0 \times 10^{9} / \mathrm{L}$, platelets $<10 \times 10^{9} / \mathrm{L}$ or $\left.\mathrm{Hb}<70 \mathrm{~g} / \mathrm{L}\right)$. 


\section{Data analysis}

From the results of the literature review, common inclusion and exclusion criteria were collated from first non-renal SLE and second LN trials to create hypothetical generalised inclusion and exclusion criteria. These criteria were then applied separately to the BILAG-BR, with patients required to meet all inclusion criteria and none of the exclusion criteria to be eligible for recruitment.

Data were entered into a secure database at the University of Manchester. Baseline demographic data are presented using descriptive statistics performed using Stata V.13 (StataCorp, College Station, Texas, USA). Differences between groups were analysed using MannWhitney $\mathrm{U}$ test for continuous data or $\chi^{2}$ test for categorical data. A logistic regression model was used to test the age-adjusted and sex-adjusted association between trial eligibility and comorbidities.

\section{RESULTS}

\section{Characteristics of RCTs included in analysis}

The systematic literature review identified 634 trials in non-renal SLE and 156 in LN; of these, 571 and 140 were excluded at abstract screening, and 51 and 10 at full-text screening, respectively. The study selection process as per the Preferred Reporting Items for Systematic Reviews and Meta-Analyses guidelines is shown in online supplemental figure 1, with our final analysis including 12 studies in non-renal SLE and 6 in LN.

\section{Inclusion criteria from non-renal SLE clinical trials}

We included the following 8 investigational medicinal products over 12 non-renal SLE clinical trials (table 1). All trials required patients to meet the ACR-1997 SLE classification criteria and required positive immunology. All included trials specified a lower age limit, usually 18 years. Disease activity for inclusion was identified by BILAG in older clinical trials, requiring $\geq 1$ BILAG-A or $\geq 2$ BILAG-B, SLEDAI-2K of at least 6 points, whereas the more contemporary clinical trials required a SELENASLEDAI score of at least 6 points. The majority of trials excluded participants taking prohibited medications including high-dose steroids $(40 \mathrm{mg}$ oral prednisolone or equivalent most frequently), recent cyclophosphamide or rituximab therapy. Three-quarters of all studies excluded severe active renal or CNS involvement and half the trials required a cut-off for proteinuria. Comorbidities were excluded in the majority of trials, either at the investigators discretion or severe previous or intercurrent illnesses, particularly those requiring steroids for treatment, chronic infections or previous cancer.

\section{Inclusion criteria from LN clinical trials}

There were six LN RCTs included from the literature review, as shown in table 2. The LUNAR, BLISS-LN and abetimus trials all required patients to meet the ACR-1997 SLE classification criteria and be antibody positive for ANA or anti-dsDNA. LN disease activity was usually specified as class III or class IV LN within 6 months to 2 years and proteinuria, most commonly with a uPCR of $100 \mathrm{mg}$ / mmol. The majority of trials required participants to be adults. Prohibited medications most frequently included previous B cell therapy, cyclophosphamide within 3 months and corticosteroid use over a given threshold.

\section{Baseline demographics of patients in BILAG-BR}

From September 2010 to June 2018, 837 patients were recruited to the BILAG-BR; 125 in the SOC cohort and 712 in the biologics cohort. The most commonly used SOC medications included mycophenolate $(n=64)$, azathioprine $(n=27)$ and cyclophosphamide $(n=24)$. Rituximab was the most common biological therapy $(n=662)$, followed by belimumab $(n=47)$. The baseline demographics of patients in the BILAG-BR are shown in the online supplemental table 1 . There were $573(68 \%)$ patients taking oral glucocorticoids and $701(87 \%)$ taking

\begin{tabular}{|c|c|c|c|c|c|c|}
\hline NCT ID & Collaborator & $\mathbf{N}$ & Phase & Completion date & Trial name & Investigational treatment \\
\hline NCT00137969 & Genentech & 262 & II/III & 2008 & EXPLORER & Rituximab \\
\hline NCT00424476 & GlaxoSmithKline & 865 & III & 2010 & BLISS-52 & Belimumab \\
\hline NCT00410384 & GlaxoSmithKline & 819 & III & 2010 & BLISS-76 & Belimumab \\
\hline NCT01196091 & Eli Lilly & 1164 & III & 2015 & ILLUMINATE 1 & Tabalumab \\
\hline NCT01205438 & Eli Lilly & 1124 & III & 2015 & ILLUMINATE 2 & Tabalumab \\
\hline NCT01262365 & UCB & 793 & III & 2015 & EMBODY 1 & Epratuzumab \\
\hline NCT01261793 & UCB & 791 & III & 2015 & EMBODY 2 & Epratuzumab \\
\hline NCT02446912 & AstraZeneca & 460 & III & 2018 & TULIP 1 & Anifrolumab \\
\hline NCT02446899 & AstraZeneca & 373 & III & 2018 & TULIP 2 & Anifrolumab \\
\hline NCT02504645 & ImmuPharma & 202 & III & 2018 & LUPUZOR & IPP-201101 \\
\hline NCT01395745 & Anthera & 442 & III & 2016 & CHABLIS SC1 & Blisibimod \\
\hline NCT00624338 & Merck & 461 & $\mathrm{II} / \mathrm{II}$ & 2012 & APRIL/SLE & Atacicept \\
\hline
\end{tabular}

N, number of participants; NCT, National Clinical Trial number; UCB, Union Chimique Belge. 


\begin{tabular}{lllllll}
\hline \multicolumn{2}{l}{ Table 2} & Included randomised clinical trials in lupus nephritis from results of literature review & \\
NCT ID & Collaborator & N & Phase & Completion date & Trial name & Investigational treatment \\
\hline NCT00282347 & Genentech & 144 & III & 2013 & LUNAR & Rituximab \\
NCT01714817 & Bristol-Myers Squibb & 695 & III & 2018 & ALLURE & Abatacept \\
NCT03021499 & Aurinia & 358 & III & 2019 & AURORA & Voclosporin \\
NCT01639339 & GlaxoSmithKline & 448 & III & 2020 & BLISS-LN & Belimumab \\
NCT00035308 & La Jolla & 330 & III & 2002 & & Abetimus \\
NCT00626197 & Roche Pharma AG & 381 & III & 2013 & BELONG LN & Ocrelizumab \\
\hline
\end{tabular}

N, number of participants; NCT, National Clinical Trial number.

antimalarials. The median daily dose of oral prednisolone was $10 \mathrm{mg}$ (IQR 7.5-20 mg). Hypertension $(24 \%, \mathrm{n}=201$ ) was the most common comorbidity followed by depression $(20 \%, \mathrm{n}=167)$, CKD $(\mathrm{n}=100,12 \%)$ and thyroid dysfunction $(\mathrm{n}=86,10 \%)$. Fifty-eight per cent of patients $(n=487)$ had one or more comorbidities.

\section{Hypothetical inclusion and exclusion from RCTs}

Overall, 307 (37\%) patients with moderate-to-severe lupus would be eligible to participate in non-renal RCTs (figure 1A), 49 (39\%) patients in the SOC cohort and $258(36 \%)$ patients in the biologics cohort. There were 166 patients with a BILAG-A in the renal domain who were designated as our 'active LN cohort'. Of these, $94(57 \%)$ would be eligible to participate in $\mathrm{LN}$ trials (figure 1B), including 81 (59\%) in the biologics group, and $13(46 \%)$ in the SOC group. The number of patients meeting each individual inclusion and exclusion criteria is shown in table 3 for non-renal lupus and table 4 for $\mathrm{LN}$, the completeness of data is demonstrated in the online supplemental tables 2 and 3 .

\section{Non-renal SLE cohort}

A total of 593 patients met all inclusion criteria, $511(72 \%)$ in the biologics group and $82(66 \%)$ in the SOC group. Fewer patients in the SOC cohort had active disease $(76 \%)$, defined as a BILAG A in 1 domain or a BILAG
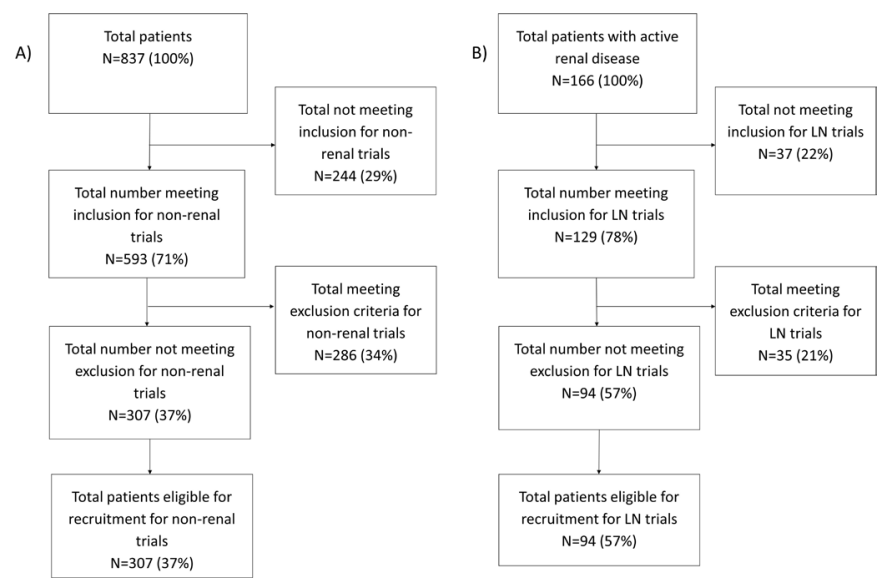

Figure 1 Flow diagram of inclusion and exclusion criteria applied to the British Isles Lupus Assessment GroupBiologics Register (BILAG-BR) for (A) non-renal and (B) lupus nephritis $(\mathrm{LN})$ clinical trials. $N$, number of participants.
B in $\geq 2$ domains, or a SLEDAI-2K $\geq 6$, compared with the biologics cohort $(87 \%)$. The most common reason for not meeting inclusion criteria was low disease activity of SLE $(\mathrm{n}=114,15 \%)$ and not meeting the ACR-1997 SLE classification criteria for SLE $(\mathrm{n}=71,9 \%)$. There were $481(86 \%)$ patients in the biologics cohort and $65(52 \%)$ in the SOC cohort that had a BILAG A or 2 BILAG B domains present; and 476 (72\%) in the biologics cohort and $72(62 \%)$ patients had a SLEDAI-2K score of 6 or

Table 3 Patients in the BILAG-BR ( $n=837)$ who did not meet the inclusion criteria or met the exclusion criteria from non-renal lupus randomised controlled trials

\begin{tabular}{|c|c|c|c|}
\hline & $\begin{array}{l}\text { Biologics } \\
(n=712)\end{array}$ & $\begin{array}{l}\text { SOC } \\
(n=125)\end{array}$ & $\mathbf{P}$ value \\
\hline \multicolumn{4}{|l|}{ Inclusion criteria } \\
\hline Age $<18$ years, $n(\%)$ & 6 & 3 & 0.127 \\
\hline $\begin{array}{l}\text { Does not meet ACR- } \\
1997 \text { SLE classification } \\
\text { criteria }\end{array}$ & 59 & 12 & 0.723 \\
\hline Negative serology & 43 & 5 & 0.323 \\
\hline Low disease activity & 86 & 28 & 0.002 \\
\hline \multicolumn{4}{|l|}{ Exclusion criteria } \\
\hline $\begin{array}{l}\text { Steroids }>40 \mathrm{mg} \\
\text { prednisolone daily }\end{array}$ & 9 & 8 & $>0.001$ \\
\hline Active CNS lupus & 39 & 7 & 0.992 \\
\hline Active renal lupus & 138 & 28 & 0.180 \\
\hline $\begin{array}{l}\text { Previous viral hepatitis } \\
\text { B or C }\end{array}$ & 20 & 1 & 0.166 \\
\hline Previous malignancy & 51 & 5 & 0.192 \\
\hline $\begin{array}{l}\text { Cyclophosphamide } \\
<90 \text { days before entry }\end{array}$ & 37 & 0 & 0.009 \\
\hline $\begin{array}{l}\text { B cell therapy }<1 \text { year } \\
\text { before entry }\end{array}$ & 50 & 0 & 0.002 \\
\hline CKD stage $4 / 5$ & 27 & 5 & 0.789 \\
\hline $\mathrm{uPCR} \geq 100 \mathrm{mg} / \mathrm{mmol}$ & 92 & 20 & 0.986 \\
\hline Low blood counts & 25 & 1 & 0.098 \\
\hline Pregnancy & 3 & 1 & 0.571 \\
\hline
\end{tabular}

ACR, American College of Rheumatology; BILAG-BR, British Isles Lupus Assessment Group-Biologics Register; CKD, chronic kidney disease; CNS, central nervous system; N, number of participants; SOC, standard of care; UPCR, urine protein-creatinine ratio. 
Table 4 Patients in the BILAG-BR with lupus nephritis who did not meet the inclusion criteria and those who met the exclusion criteria from renal lupus randomised controlled trials

\begin{tabular}{|c|c|c|c|}
\hline & $\begin{array}{l}\text { Biologics } \\
(n=138)\end{array}$ & $\begin{array}{l}\text { SOC } \\
(n=28)\end{array}$ & $P$ value \\
\hline \multicolumn{4}{|l|}{ Inclusion criteria } \\
\hline Age $<18$ years & 2 & 2 & 0.099 \\
\hline $\begin{array}{l}\text { Does not meet ACR- } \\
1997 \text { SLE classification } \\
\text { criteria }\end{array}$ & 4 & 4 & 0.013 \\
\hline Negative serology & 3 & 2 & 0.183 \\
\hline uPCR $<100 \mathrm{mg} / \mathrm{mmol}$ & 8 & 4 & 0.294 \\
\hline \multicolumn{4}{|l|}{ Exclusion criteria } \\
\hline $\begin{array}{l}\text { Steroids }>40 \mathrm{mg} \\
\text { prednisolone daily }\end{array}$ & 4 & 5 & 0.003 \\
\hline $\begin{array}{l}\text { Active CNS lupus (BILAG } \\
\text { A) }\end{array}$ & 4 & 1 & 0.811 \\
\hline $\begin{array}{l}\text { Previous viral hepatitis } \\
\text { B or C }\end{array}$ & 4 & 0 & 0.337 \\
\hline Previous malignancy & 7 & 2 & 0.659 \\
\hline $\begin{array}{l}\text { Cyclophosphamide } \\
<90 \text { days before entry }\end{array}$ & 8 & 0 & 0.192 \\
\hline $\begin{array}{l}\text { B cell therapy }<1 \text { year } \\
\text { before entry }\end{array}$ & 6 & 0 & 0.261 \\
\hline CKD stage $4 / 5$ & 19 & 3 & 0.532 \\
\hline Pregnancy & 0 & 1 & 0.026 \\
\hline
\end{tabular}

ACR, American College of Rheumatology; BILAG-BR, British Isles Lupus Assessment Group-Biologics Register; CKD, chronic kidney disease; CNS, central nervous system; N.S., non-significant; SOC, standard of care; UPCR, urine protein-creatinine ratio.

greater. Of the patients meeting all inclusion criteria, a total of $286(48 \%)$ patients met at least one exclusion criteria, and of these $124(40 \%)$ met two or more exclusion. The most common causes for exclusion were active renal SLE, a uPCR $>100 \mathrm{mg} / \mathrm{mmol}$ or a previous history of malignancy. Ineligible patients had shorter median (IQR) disease duration compared with eligible patients: $2.9(0.5-8.9)$ years vs $5.1(1.5-11.3)$ years $(\mathrm{p}<0.01)$, respectively. Ineligible patients did not differ in age $(\mathrm{p}=0.8)$, sex $(\mathrm{p}=0.7)$ or ethnicity $(\mathrm{p}=0.7)$.

\section{Renal SLE cohort}

In the biologics and SOC groups, respectively, 112 $(81 \%)$ and $17(61 \%)$ met all inclusion criteria. A higher percentage of patients in the biologics group (97\%) met the ACR-1997 classification criteria for SLE, compared with the SOC group (85\%). Other inclusion parameters, including age $\geq 18$ years, presence of positive serology and a UPCR $\geq 100 \mathrm{mg} / \mathrm{mmol}$ were similar between the biologics and SOC cohorts. Five patients (4\%) with active renal disease did not have positive serology and therefore would have been excluded from RCTs.
The renal SLE trial exclusion criteria disqualified 39 $(28 \%)$ patients in the biologics group and $10(36 \%)$ patients in the SOC group. Of those who met all inclusion criteria, there were $10(8 \%)$ patients who met two or more exclusion criteria. The most commonly fulfilled exclusion criteria were existing renal impairment $(n=22$, $14 \%)$, high background steroid use $(\mathrm{n}=9,6 \%)$, history of malignancy $(\mathrm{n}=9,5 \%)$ and prior cyclophosphamide use $(n=8,5 \%)$. The percentage of patients whose steroid dose was over $40 \mathrm{mg}$ equivalent per day was higher in the SOC group compared with the biologics group. Ineligible patients did not differ from eligible patients according to disease duration $(\mathrm{p}=0.1)$, age $(\mathrm{p}=0.3)$, sex $(\mathrm{p}=0.2)$ or ethnicity $(\mathrm{p}=0.1)$.

\section{Comorbidities between non-renal and renal groups}

The active renal disease cohort (BILAG renal A, $\mathrm{n}=166$ ) had higher incidence rates of hypertension ( $34 \%$ vs $22 \%$, $\mathrm{p}=0.001)$ and pre-existing CKD stage 4 or $5(14 \%$ vs $2 \%$, $\mathrm{p}<0.001)$ compared with those who did not have active renal disease (no BILAG renal A, $\mathrm{n}=671$ ).

\section{Comorbidities in patients included or excluded from clinical trials}

There were a lower number of comorbidities in patients eligible for recruitment to both types of trials. In patients with non-renal SLE, 51\% $\quad(n=158 / 307)$ of eligible patients had one or more comorbidity, compared with $62 \%(n=329 / 530)$ of ineligible subjects. Three or more comorbidities were present in $14.2 \% \quad(\mathrm{n}=75)$ of patients ineligible, compared with $9.4 \% \quad(n=29)$ of those eligible to participate. The number of patients with $\geq 1$ comorbidity was similar in the $\mathrm{LN}$ cohort between those eligible and ineligible (62\% vs $61 \%)$. In an age-adjusted and sexadjusted analysis, eligibility for trial inclusion was associated with fewer comorbidities (non-renal: OR 0.71, $95 \%$ CI 0.56 to 0.88 ).

\section{DISCUSSION}

Despite unprecedented drug development in SLE in recent years, the standard of care remains limited, with a number of medications given off-label without supporting evidence from large double-blinded RCTs. Just two drugs, belimumab (SLE and LN) and voclosporin (LN only), have been approved in over 50 years, however, these are not effective in all patients with SLE and do not consistently induce full disease remission in the majority of patients. ${ }^{9}{ }^{12}$ In recent years, success has been seen with both phase II and phase III trials including anifrolumab (TULIP2) in non-renal SLE. ${ }^{7-9} 17-20$

RCTs represent the most scientifically rigorous method of hypothesis testing available and are considered the gold standard for evaluating the effectiveness of interventions. Clinical trials show what can be accomplished under controlled, restricted and carefully observed conditions; however, this will not necessarily be observed when a medicine is authorised for general use. 
This study is the first to compare a large national registry of patients with SLE with moderate-to-severe disease, the BILAG-BR, to major published eligibility criteria from RCTs in non-renal SLE and LN. We have shown that $63 \%$ of patients with non-renal SLE and $43 \%$ of patients with LN would not be eligible to participate in an RCT. These results indicate that phase III clinical trials results may only partially reflect real-world SLE cohorts with similar levels of disease activity. When interpreting the results of clinical trials, caution should be paid in reviewing whether all patients have been adequately represented in the clinical trial.

Patient safety must be paramount in clinical trials. This study uses inclusion and exclusion criteria from phase III trials which focus on proving efficacy for licencing, and confirming the safety profile from phase II trials in a broader population. Clinical trials are notoriously difficult to recruit due to the low abundance of participants in the outpatient lupus population. This study highlights the ability to improve clinical trial recruitment through the aggregation of marginal gains. There is wide variation in acceptable exclusion criteria (and individual thresholds of each, eg, estimated glomerular filtration rate, time from previous anti-CD20 therapy, etc). Therefore, each criterion needs to be critically reviewed to ensure it is safeguarding participants' safety, and not at the expense of reducing recruitment potential and thus the external validity of the trial. This must be done with careful reference to the mechanism of action of the investigational drug.

This analysis reported no significant difference in the ethnicity of UK patients eligible for RCTs based on common published inclusion and exclusion criteria. Previous studies have reported that real-world participation in SLE clinical trials does vary by ethnicity, suggesting external factors responsible for the lack of representation of ethnic minorities in clinical research. ${ }^{21}$ These may include both patient barriers (such as language) to participation and clinician barriers in referring patients for consideration ${ }^{22}$; the Lupus Foundation of America is presently undertaking work with the aim of increasing awareness of SLE research in under-represented populations and the ACR's lupus initiative is dedicated to reducing health inequalities among disproportionately affected populations. This is important as low participation in SLE RCTs from ethnic minority groups results in a lack of data on the effectiveness and safety of treatments in those who have the highest morbidity and mortality from SLE. ${ }^{23-25}$ More diverse recruitment strategies, with an emphasis on hard-to-reach populations, will lead to better generalisability of results.

Our literature review showed that non-renal SLE and the majority of $\mathrm{LN}$ trials required patients to be defined using the ACR-1997 SLE classification criteria and be seropositive for an autoantibody at the time of recruitment. Classification criteria are used in clinical trials to identify a well-defined population of patients with high specificity for SLE, however, they are not diagnostic criteria and may exclude a significant proportion of patients. ${ }^{26} \mathrm{In}$ both renal and non-renal trials, recent seropositivity was frequently required for inclusion, which would result in $6 \%$ of patients in BILAG-BR being excluded from clinical trials. A secondary analysis of the phase II belimumab trial had noted greater efficacy for belimumab in patients with SLE with a positive ANA or anti-dsDNA antibody at trial entry. ${ }^{27}$ The phase III BLISS trial programme carried forward this entry criteria which is now standard across most other SLE trials with these tests routinely tested centrally prior to recruitment to clinical trials. ${ }^{6}{ }^{62}$ Whether active serology at trial entry is necessary for all SLE trials, or only for B-cell targeted therapy remains to be demonstrated. If these criteria were removed more patents in our cohorts would theoretically be eligible.

Clinical trial recruitment is challenging due to the low abundance of patients in the outpatient lupus population. In the years 2018-2020, recruitment to BILAG-BR from over 60 UK centres was on average 16 patients per month. Results from this analysis show that only $37 \%$ of patients with moderate-to-severe SLE would be eligible for recruitment to non-renal clinical trials. Across the UK, this would translate to six patients per month being eligible for clinical trials. There is therefore a limit to the number of lupus trials that potentially could be open in a country such as the UK at any given time. To date, 62 centres have contributed to the BILAG-BR, however, the top 10 recruiting centres account for $67 \%$ (962/1435) of all patients recruited. It is important that patients with active lupus are managed in collaboration with a large centre so that patients can be recruited to clinical trials.

Currently, there are many trials recruiting globally, with eight RCTs (including six phase II and two phase III) in SLE actively recruiting in the UK (ClinicalTrials. gov; December 2020). As every individual inclusion and exclusion criteria impacts patient recruitment, careful scrutiny is needed before adoption. Our data suggest that exclusion criteria should be minimised where safe to do so, and phase III protocols should be 'forgiving' to maximise recruitment potential and speed their conduct.

Certain SLE disease manifestations are excluded in trials to improve patient safety, such as CNS involvement in SLE which has high morbidity. We found that $46(6 \%)$ patients would be excluded due to active CNS SLE. As RCTs exclude these patients, there is no high-quality evidence for their management, and dedicated trials in CNS lupus are needed.

This study showed that in non-renal clinical trials, patients excluded from participating in clinical trials had a cumulatively larger mean number of comorbidities than those included. Similar studies have been carried out in the field of oncology with chemotherapy trials being more likely to exclude patients with severe disease such as CNS metastasis, comorbidities and history of other malignancies. ${ }^{28}{ }^{29}$ Despite this, excluding the patients who may be more likely to represent the population treated in clinical settings leaves patients susceptible to unintended harm from inappropriate generalisation of trial results. One 
solution may be to have less stringent limits on comorbidities at recruitment where safe to do so, with stratification of recruitment groups based on comorbidities. We would advocate a focused approach to this, with careful scrutiny of the mechanism of action of the experimental drug. For example, allowing levels of CKD in therapies with no known renal toxicity concerns.

An additional challenge for drug development is that patients with SLE are treated with corticosteroids and other immunosuppressive medications, which cannot be discontinued on joining an RCT. This increases the placebo response rate in trials, which can obscure the effects of trial medications, particularly if they are only moderately efficacious. Most studies excluded patients that had received rituximab within a year or cyclophosphamide in the previous 6 months due to their long-lasting effects. This results in patients' refractory to these medications being excluded from clinical trials and potentially efficacious treatment, further reducing the trial population. Suggested mitigations of this include measurement of B-cell reconstitution prior to enrolment. There is also wide variation in corticosteroid dosing by clinicians, some of which is driven by patient-independent factors. ${ }^{30} \mathrm{We}$ would suggest a focus in the early phase of the trial to allow a protocol-led harmonisation and standardisation of steroid dosing.

This study does have limitations. Complete data were not available for every patient in this study. We assessed the amount of people who did not meet all inclusion criteria or who met an exclusion criteria. Employing this methodology may have led to a conservative estimate of patients who would be ineligible for clinical trial participation. It is unlikely that data are 'missing at random' and for variables where there is a high percentage of missing data, such as uPCR, this likely represents a bias to those without an available measurement being more likely to have a normal result.

Additionally, many of the clinical trials that formed our eligibility criteria excluded patients at the discretion of the principle study investigator, such as those with 'serious intercurrent illness' or conditions requiring significant steroid use. It is difficult to accurately conclude which patients in BILAG-BR would be deemed ineligible on this basis. Other eligibility criteria from other trial protocols were not included in this study, such as no recent live vaccination, no recent dose adjustments in background medications and no investigational biological agents. By not being able to fully describe the nuance of eligibility criteria in clinical trials, this study is likely to underestimate the number of patients not eligible for phase III clinical trials.

A further limitation is that the SLICC 2012 criteria for SLE may be used in lieu of the ACR-1997 criteria in the classification of SLE in some clinical trials. BILAG-BR started prior to these being published, therefore data on SLICC criteria are not complete in the BILAG-BR and have not been presented. ${ }^{31}$

\section{CONCLUSION}

This is the first study to compare a national registry of patients with moderate-to-severe SLE to major published RCTs in SLE and LN. We found that nearly two-thirds of patients with moderate-to-severe non-renal SLE and almost a half of patients with active LN would not be eligible for participation in clinical trials. These results indicate that phase III clinical trial results may only partially reflect real-world SLE cohorts. When interpreting the results of these clinical trials, caution should be paid in reviewing whether the patient has been adequately represented in the clinical trial.

\section{Twitter Sarah Dyball @sarahdyball1 and lan N Bruce @Lupusdoc}

Acknowledgements BILAG-BR collaborators-Dr Hazem Youssef, Aberdeen Royal Infirmary; Professor David Jayne, Addenbrooke's Hospital; Dr Liza Mccann, Alder Hey Children's Hospital; Dr Anurag Bharadwaj, Basildon Hospital; Dr Ed Vital, Chapel Allerton Hospital; Professor Caroline Gordon, City Hospital Birmingham, Sandwell and West Birmingham Hospitals NHS Trust; Dr Deepti Kapur, Countess of Chester Hospital; Professor Chee-Seng Yee, Doncaster Royal Infirmary; Dr Bridget Griffiths, Freeman Hospital Newcastle; Dr Laith Al Sweedan, George Eliot Hospital NHS Trust; Dr David D'Cruz, Guy's Hospital; Dr Asad Zoma, Hairmyres Hospital; Dr Erin Vermaak, Haywood Hospital; Dr Francesco Carlucci, Imperial College Hospital; Dr Richard Watts, Ipswich Hospital; Dr Patrick Gordon, King's College Hospital; Dr Shireen Shaffu, Leicester Royal Infirmary; Dr Jananath Wijeyekoon, Lister Hospital; Dr Zoe Mclaren, Liverpool University Hospitals NHS Foundation Trust; Dr Mike Batley, Maidstone Hospital; Dr Luke Gompels, Musgrove Park Hospital; Dr T Sheeran, New Cross Hospital and Cannock Chase Hospital; Dr Chetan Mukhtyar, Norfolk and Norwich University Hospital; Dr Rachel Jeffery, Northampton General Hospital; Dr Shahir Hamdulay, Northwick Park Hospital; Dr Fouz Rahmeh, Poole Hospital NHS Foundation Trust; Dr Steven Young Min, Queen Alexandra Hospital Portsmouth; Dr Ben Rhodes, Queen Elizabeth Hospital Birmingham; Dr Denise De Lord, Queen Elizabeth the Queen Mother Hospital, Margate; Dr Peter Lanyon, Queen's Medical Centre, Nottingham; Dr Antoni Chan, Royal Berkshire Hospital; Professor Lee-Suan, The Royal Blackburn Hospital; Dr Jonathan Marks, Royal Bournemouth and Christchurch Hospitals NHS Foundation Trust; Dr David Hutchinson, Royal Cornwall Hospital; Dr Marian Regan, Royal Derby Hospital; Dr Richard Haigh, Royal Devon and Exeter Hospital; Dr Richard Stratton, Royal Free Hospital; Dr Ceril Rhys-Dillon, Royal Glamorgan Hospital; Dr Mohamed Akil, Royal Hallamshire Hospital; Dr Devesh Mewar, Royal Liverpool and Broadgreen Hospital; Dr Sarah Skeoch, Royal United Hospitals Bath; Dr Nicola Erb Russells Hall Hospital; Dr Edmond O'Riordan, Salford Royal NHS Trust; Dr Sarah Bartram, Salisbury District Hospital; Dr Mary Gayed, Solihull Hospital; Dr Bhaskar Dasgupta, Southend Hospital NHS Trust; Dr Harsha Gunwardena, Southmead Hospital Bristol; Dr Dev Pyne, St Bartholomew's Hospital; Dr Arvind Kaul, St George's Hospital; Dr V E Abernethyst, Helens and Knowsley Teaching Hospitals NHS Trust; Dr Bhrigu Raj Sood, St Helier Hospital; Dr Mike Batley, Tunbridge Wells Hospital; Professor David Isenberg, University College Hospital; Dr Nicola Gullick, University Hospital Coventry \& Warwickshire; Professor Christopher Edwards, University Hospital Southampton; Dr Joanna C Robson, University Hospitals Bristol NHS Foundation Trust; Dr Jon King, University Hospitals Plymouth NHS Trust; Dr Adrian Farrell, Worcestershire Acute Hospitals; Dr Sahena Haque, Wythenshawe Hospital; Dr Sally Knights, Yeovil District Hospital.

Contributors SD, SC, ES, EMMcC, INB and BP contributed to the design of the study. SD and SC completed the literature review of clinical trials in SLE. SD, SC and ES have analysed the data and performed statistical analyses. All authors have discussed the results and contributed to the writing of the manuscript and approved the final manuscript.

Funding The BILAG-BR acknowledges funding from Lupus UK, Roche and GSK. SD is an MRC Clinical Training Fellow based at the University of Manchester supported by the North West England Medical Research Council Fellowship Scheme in Clinical Pharmacology and Therapeutics, which is funded by the Medical Research Council (Award Ref. MR/N025989/1), Roche Pharma, Eli Lilly and Company Limited, UCB Pharma, Novartis, the University of Liverpool and the University of Manchester. INB is a National Institute for Health Research (NIHR) Senior Investigator and is funded by the NIHR Manchester Biomedical Research Centre. BP is supported by the NIHR Manchester Biomedical Research Centre and NIHR Manchester Clinical Research Facility. 
Disclaimer The views expressed in this publication are those of the author(s) and not necessarily those of the NHS, the NIHR or the Department of Health.

Competing interests INB has received grant support from Genzyme/Sanofi, GSK, Roche and UCB; consulting fees from AstraZeneca, Eli Lilly, GSK, Merck Serono, UCB and ILTO0; and was a speaker for AstraZeneca, GSK and UCB. BP has received grant support from Genzyme/Sanofi and GSK, honoraria from Fresenius-Kabi and AbbVie and was a speaker for Eli Lilly and Roche.

Patient consent for publication Not required.

Ethics approval Ethics approval was granted by the NRES Committee North West-Greater Manchester West (REC: 09/H1014/64) and the local Research and Development departments at participant sites. Health Research Authority dated 9 November 2009 (IRAS ref. 24407). All patients provided written informed consent at the time of study registration.

Provenance and peer review Not commissioned; externally peer reviewed. Data availability statement No data are available. The British Isles Lupus Assessment Group-Biologics Register is an ongoing prospective study and no data are available.

Supplemental material This content has been supplied by the author(s). It has not been vetted by BMJ Publishing Group Limited (BMJ) and may not have been peer-reviewed. Any opinions or recommendations discussed are solely those of the author(s) and are not endorsed by BMJ. BMJ disclaims all liability and responsibility arising from any reliance placed on the content. Where the content includes any translated material, BMJ does not warrant the accuracy and reliability of the translations (including but not limited to local regulations, clinical guidelines, terminology, drug names and drug dosages), and is not responsible for any error and/or omissions arising from translation and adaptation or otherwise.

Open access This is an open access article distributed in accordance with the Creative Commons Attribution Non Commercial (CC BY-NC 4.0) license, which permits others to distribute, remix, adapt, build upon this work non-commercially, and license their derivative works on different terms, provided the original work is properly cited, appropriate credit is given, any changes made indicated, and the use is non-commercial. See: http://creativecommons.org/licenses/by-nc/4.0/.

\section{ORCID iDs}

Sarah Dyball http://orcid.org/0000-0001-6201-7391

lan N Bruce http://orcid.org/0000-0003-3047-500X

\section{REFERENCES}

1 Cervera R, Khamashta MA, Font J, et al. Morbidity and mortality in systemic lupus erythematosus during a 10-year period: a comparison of early and late manifestations in a cohort of 1,000 patients. Medicine 2003;82:299-308.

2 Danila MI, Pons-Estel GJ, Zhang J, et al. Renal damage is the most important predictor of mortality within the damage index: data from LUMINA LXIV, a multiethnic US cohort. Rheumatology 2009;48:542-5.

3 Mok CC, Kwok RCL, Yip PSF. Effect of renal disease on the standardized mortality ratio and life expectancy of patients with systemic lupus erythematosus. Arthritis Rheum 2013;65:2154-60.

4 Arriens C, Polyakova S, Adzerikho I, et al. OP0277 AURORA phase 3 study demonstrates voclosporin statistical superiority over standard of care in lupus nephritis (LN). Ann Rheum Dis 2020;79:172.2-3.

5 Merrill JT, van Vollenhoven RF, Buyon JP, et al. Efficacy and safety of subcutaneous tabalumab, a monoclonal antibody to B-cell activating factor, in patients with systemic lupus erythematosus: results from ILLUMINATE-2, a 52-week, phase III, multicentre, randomised, double-blind, placebo-controlled study. Ann Rheum Dis 2016;75:332-40.

6 Navarra SV, Guzmán RM, Gallacher AE, et al. Efficacy and safety of belimumab in patients with active systemic lupus erythematosus: a randomised, placebo-controlled, phase 3 trial. Lancet 2011;377:721-31.

7 Furie RA, Morand EF, Bruce IN, et al. Type I interferon inhibitor anifrolumab in active systemic lupus erythematosus (TULIP-1): a randomised, controlled, phase 3 trial. Lancet Rheumatol 2019;1:e208-19.

8 Morand EF, Furie R, Tanaka Y, et al. Trial of Anifrolumab in active systemic lupus erythematosus. N Engl J Med 2020;382:211-21.
9 Furie R, Rovin BH, Houssiau F, et al. Two-Year, randomized, controlled trial of belimumab in lupus nephritis. $N$ Engl $\mathrm{J}$ Med 2020;383:1117-28.

10 Isenberg DA, Petri M, Kalunian K, et al. Efficacy and safety of subcutaneous tabalumab in patients with systemic lupus erythematosus: results from ILLUMINATE-1, a 52-week, phase III, multicentre, randomised, double-blind, placebo-controlled study. Ann Rheum Dis 2016;75:323-31.

11 Clowse MEB, Wallace DJ, Furie RA, et al. Efficacy and safety of Epratuzumab in moderately to severely active systemic lupus erythematosus: results from two phase III randomized, double-blind, placebo-controlled trials. Arthritis Rheumatol 2017;69:362-75.

12 Furie R, Petri M, Zamani O, et al. A phase III, randomized, placebocontrolled study of belimumab, a monoclonal antibody that inhibits B lymphocyte stimulator, in patients with systemic lupus erythematosus. Arthritis Rheum 2011;63:3918-30.

13 Touma Z, Gladman DD. Current and future therapies for SLE: obstacles and recommendations for the development of novel treatments. Lupus Sci Med 2017;4:e000239.

14 Isenberg DA, Rahman A, Allen E, et al. BILAG 2004. development and initial validation of an updated version of the British Isles lupus assessment group's disease activity index for patients with systemic lupus erythematosus. Rheumatology 2005;44:902-6.

15 Gao Z, Ni D, Li F. [The normal value and clinical applications of the blink reflex]. Zhonghua Er Bi Yan Hou Ke Za Zhi 1998;33:347-9.

16 Hochberg MC. Updating the American College of rheumatology revised criteria for the classification of systemic lupus erythematosus. Arthritis Rheum 1997;40:1725.

17 Wallace DJ, Furie RA, Tanaka Y, et al. Baricitinib for systemic lupus erythematosus: a double-blind, randomised, placebo-controlled, phase 2 trial. Lancet 2018;392:222-31.

18 van Vollenhoven RF, Hahn BH, Tsokos GC, et al. Efficacy and safety of ustekinumab, an IL-12 and IL-23 inhibitor, in patients with active systemic lupus erythematosus: results of a multicentre, double-blind, phase 2, randomised, controlled study. Lancet 2018;392:1330-9.

19 Arriens C, Polyakova S, Adzerikho I, et al. OP0277 Aurora phase 3 study demonstrates Voclosporin statistical superiority over standard of care in lupus nephritis (LN). Ann Rheum Dis 2020;79:172.2-3.

20 Hermann V, Batalov A, Smakotina S, et al. First use of cenerimod, a selective $\mathrm{S}_{1 \mathrm{P}}$ receptor modulator, for the treatment of SLE: a double-blind, randomised, placebo-controlled, proof-of-concept study. Lupus Sci Med 2019;6:e000354.

21 Falasinnu T, Chaichian Y, Bass MB, et al. The representation of gender and Race/Ethnic groups in randomized clinical trials of individuals with systemic lupus erythematosus. Curr Rheumatol Rep 2018;20:20

22 Sheikh SZ, Wanty NI, Stephens J, et al. The state of lupus clinical trials: minority participation needed. J Clin Med 2019;8:1245.

23 Korbet SM, Schwartz MM, Evans J, et al. Severe lupus nephritis: racial differences in presentation and outcome. J Am Soc Nephrol 2007;18:244-54

24 Bernatsky S, Boivin J-F, Joseph L, et al. Mortality in systemic lupus erythematosus. Arthritis Rheum 2006;54:2550-7.

25 Zhang F, Bae S-C, Bass D, et al. A pivotal phase III, randomised, placebo-controlled study of belimumab in patients with systemic lupus erythematosus located in China, Japan and South Korea. Ann Rheum Dis 2018;77:355-63.

26 Aringer M, Costenbader K, Daikh D, et al. 2019 European League against Rheumatism/American College of rheumatology classification criteria for systemic lupus erythematosus. Ann Rheum Dis 2019;78:1151-9.

27 Stohl W, Hiepe F, Latinis KM, et al. Belimumab reduces autoantibodies, normalizes low complement levels, and reduces select B cell populations in patients with systemic lupus erythematosus. Arthritis Rheum 2012;64:2328-37.

28 Duma N, Kothadia SM, Azam TU, et al. Characterization of comorbidities limiting the recruitment of patients in early phase clinical trials. Oncologist 2019;24:96-102.

29 Unger JM, Hershman DL, Fleury ME, et al. Association of patient comorbid conditions with cancer clinical trial participation. JAMA Oncol 2019;5:326-33.

30 Little J, Parker B, Lunt M, et al. Glucocorticoid use and factors associated with variability in this use in the systemic lupus international collaborating clinics inception cohort. Rheumatology 2018;57:677-87.

31 Petri M, Orbai A-M, Alarcón GS, et al. Derivation and validation of the systemic lupus international collaborating clinics classification criteria for systemic lupus erythematosus. Arthritis Rheum 2012;64:2677-86. 